\title{
Malignant peripheral nerve sheath tumor associated with neurofibromatosis type 1, with metastasis to the heart: a case report
}

\author{
Masanori Kitamura', Naoki Wada², Shigenori Nagata', Norishige lizuka', Yu-Fen Jin', Miki Tomoeda', Michiko Yuki', \\ Norifumi Naka ${ }^{3}$, Nobuhito Araki $^{3}$, Chikao Yutani ${ }^{4}$, Yasuhiko Tomita ${ }^{1 *}$
}

\begin{abstract}
A rare case is presented of a 61-year-old man with a malignant peripheral nerve sheath tumor associated with neurofibromatosis type 1, with metastasis to the heart. The primary tumor originated in the right thigh in 1982. Since then, the patient has had repeated local recurrences in spite of repeated surgical treatment and adjuvant chemotherapy. He has developed previous metastases of the lung and heart. The patient died of cardiac involvement.
\end{abstract}

\section{Background}

Malignant peripheral nerve sheath tumor (MPNST) is an aggressive and uncommon neoplasm that develops within a peripheral nerve; most cases of which are associated with neurofibromatosis type 1 (NF1). Metastasis of MPNST usually occurs in the lung [1], whereas cardiac metastasis of MPNST is quite rare [2-4]. In this report, we describe a case of MPNST that metastasized to the heart, with a review of the literature.

\section{Case Presentation}

A 61-year-old man presented with clinical stigmata of NF1. Genetic analysis had not been performed. He had no family history and had developed multiple neurofibromas and café-au-lait spots on his body (Figure 1). He underwent surgical treatment for a growing tumor (details are unknown) on the posterior surface of his right thigh at another hospital in April 1982, and was referred to our institution because of regrowth of the tumor in October 1990. Wide resection was performed in November 1990 (Figure 2). The histological diagnosis was MPNST developed on a background of NF1, and he received adjuvant chemotherapy. Since 1990, the patient has had repeated local recurrences (7 times, until May 2003) and has received surgical treatment for each

\footnotetext{
* Correspondence: yasuhiko-tomita@umin.ac.jp

'Department of Pathology, Osaka Medical Center for Cancer and

Cardiovascular Diseases, 1-3-3 Nakamichi, Higashinari, Osaka, 537-8511, Japan
}

recurrent tumor. In December 2003, the patient presented with a skin tumor of the head, and the lesion was resected. The histological diagnosis was MPNST that arose in a neurofibroma of the head skin. Two years later, the right leg was amputated at the hip joint because of local recurrence of the leg lesion, with aggressive growth and rupture. Computed tomography (CT) performed in August 2005 showed multiple lung lesions, suggestive of tumor metastases. Some of the lung lesions were resected surgically in January 2006 and diagnosed histologically as MPNST metastases. After the operation, he developed new lung metastases. In May 2007, he was admitted to our institution with sudden dyspnea and palpitations. Echocardiography revealed an enlarged heart and cardiac effusion, which did not suggest cardiac tamponade. Magnetic resonance imaging showed the presence of a large tumor $(9 \mathrm{~cm})$ in the inferior-posterior wall of the heart (Figure 3). CT revealed an infarction of the inferior lobe of the left lung, which was derived from tumor emboli. As a result of the worsening condition, irradiation was applied to the cardiac mass $(2 \times 25$ Gy). The patient's condition still worsened, with increasing cardiac effusion and arrhythmia. Regardless of drainage of the effusion, he died of acute heart failure soon after. At autopsy, the cardiac mass was confirmed histologically as MPNST (Figure 4). The cardiac lesion was thought to be a metastatic tumor, because neurofibroma was not seen as 

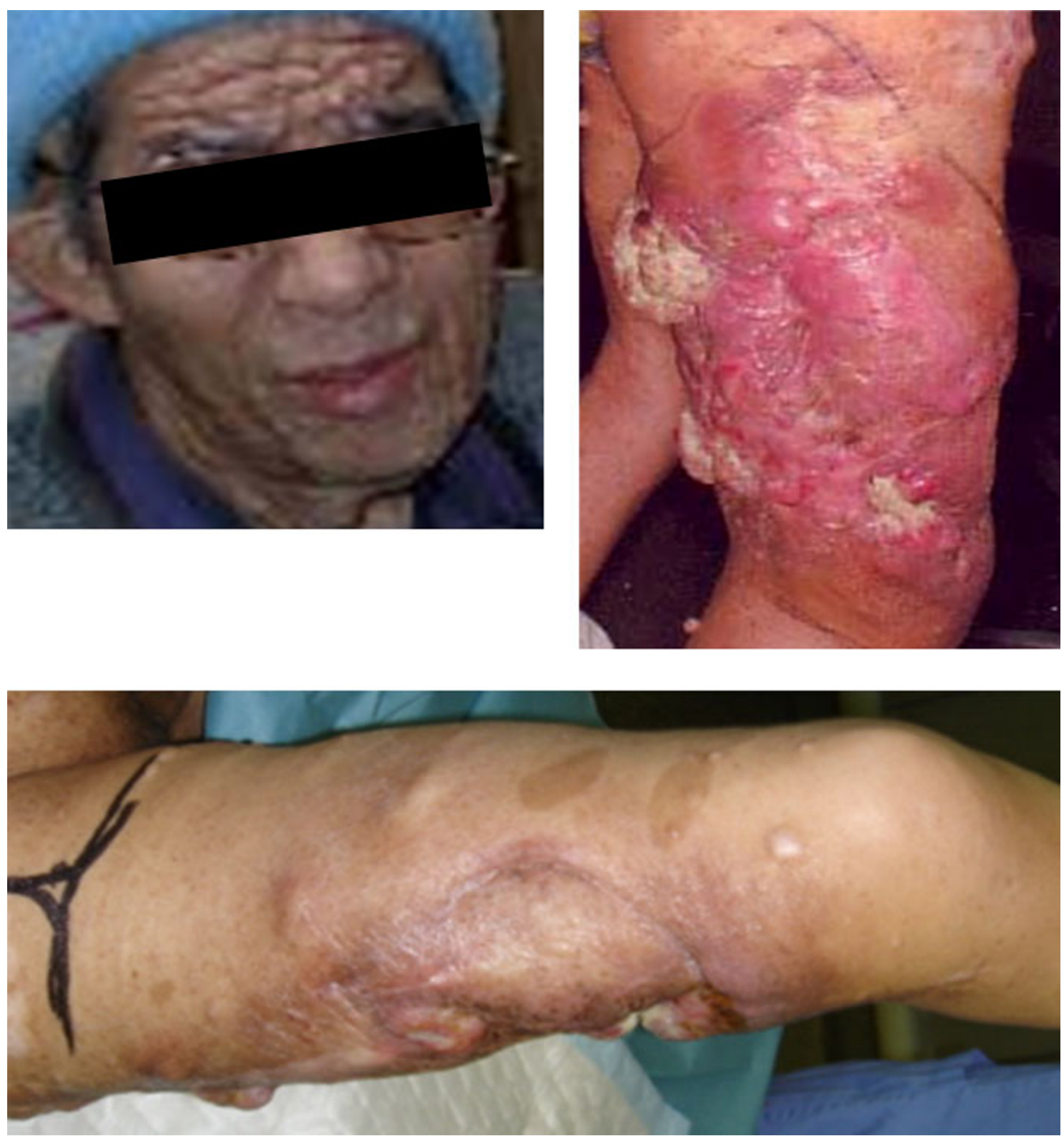

Figure 1 Clinical manifestations of NF1: multiple cutaneous neurofibromas and café-au-lait spots

possible precursor within the lesion. Serial sections suggested that the tumor involved the conduction system. MPNST affected both lungs. Histological images of cardiac and pulmonary tumor were similar to those of the tumor of the right leg that was amputated in 2005 (Figure 2).

\section{Discussion}

Patients with NF are at greatest risk for developing sarcomas, including MPNST. The incidence of MPNST arising in NF is $4.6 \%$, which is much higher than the $0.001 \%$ in general population [5]. The most common metastatic site of MPNST is the lung [1,4]. Cardiac involvement from metastatic MPNST is extremely rare, whether with or without a background of NF [2-4]. Our patient is one of the extremely rare cases of cardiac metastasis of MPNST associated with NF1. Most of the cardiac metastases are preceded by other metastatic lesions, such as in the lung. Although the possibility of cardiac involvement becomes higher as the tumor progresses, details of the histopathological features specific to cardiac metastasis remain to be investigated. Recent studies have in part revealed the genomic imbalance in sporadic and NF1-associated MPNST [6,7]. The biology 
A

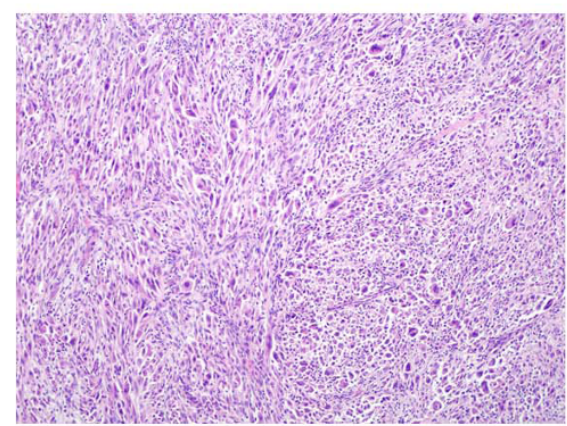

C

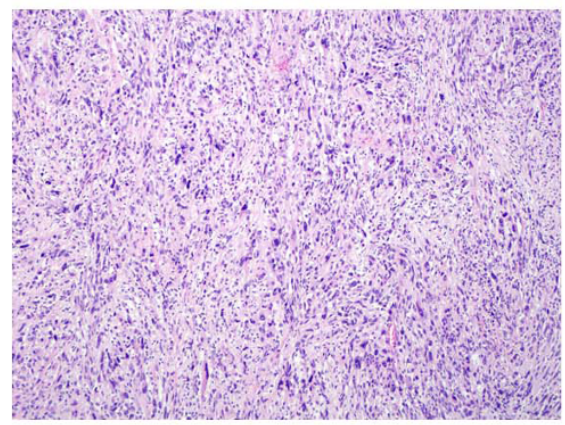

E

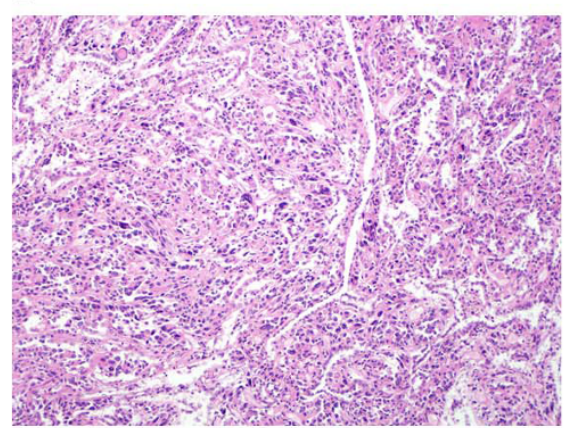

G

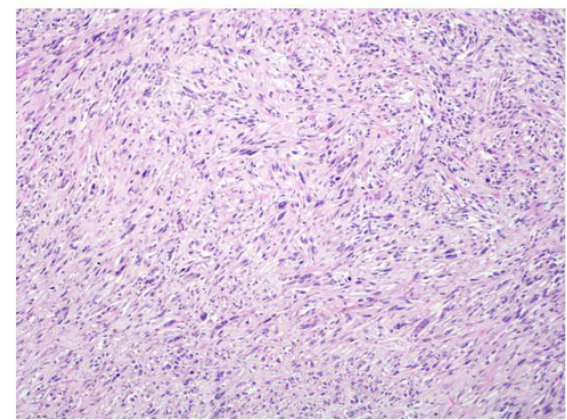

B

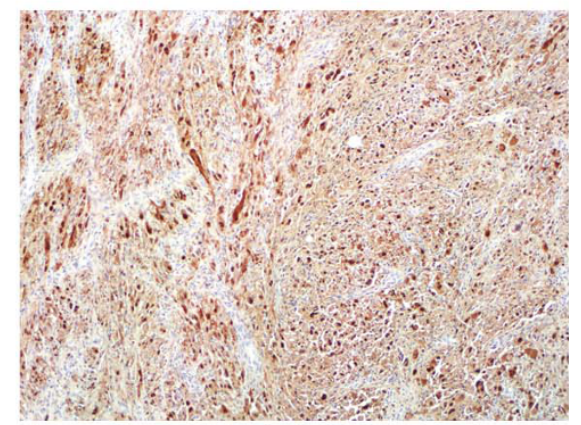

D

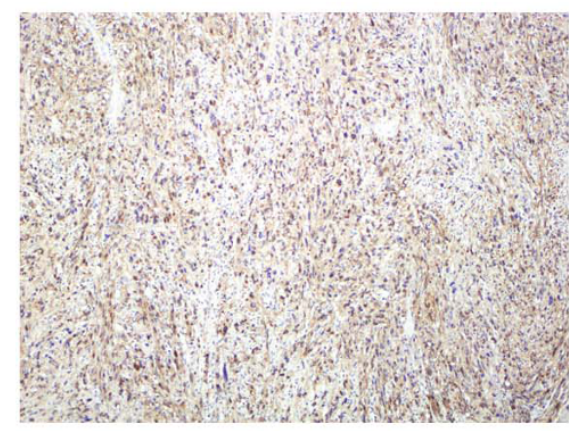

F

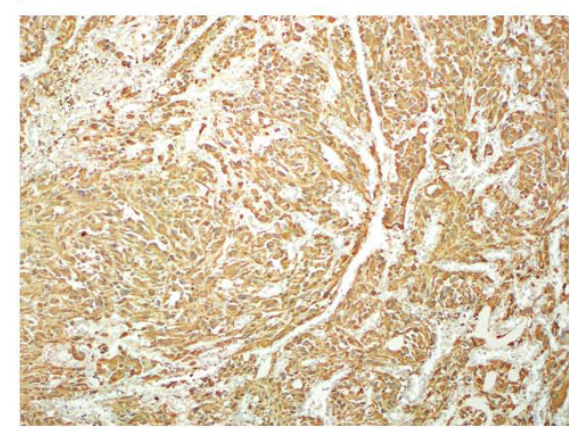

$\mathrm{H}$

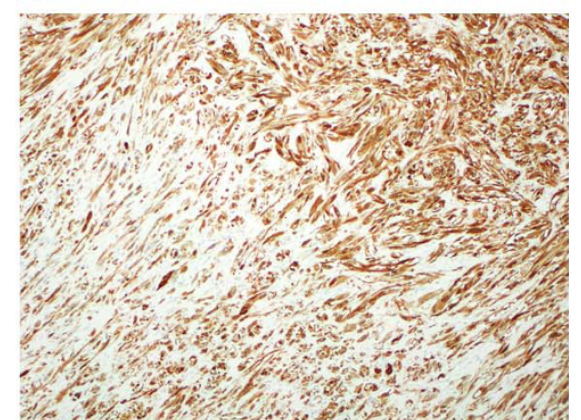

Figure 2 Hematoxylin and eosin and S-100 staining of the tumors of the thigh treated surgically in 1990 (A, B), recurrent tumor operated in 1995 (C, D) and metastatic tumor in heart (E, F) and lung (G, H) at autopsy in 2007. A, C, E, G: Hematoxylin and eosin staining; B, D, F, H: S-100 immunohistochemistry. Histological factors such as cellularity, degree of nuclear atypia, mitotic counts, and S-100 immunoreactivity were similar among the specimens. 


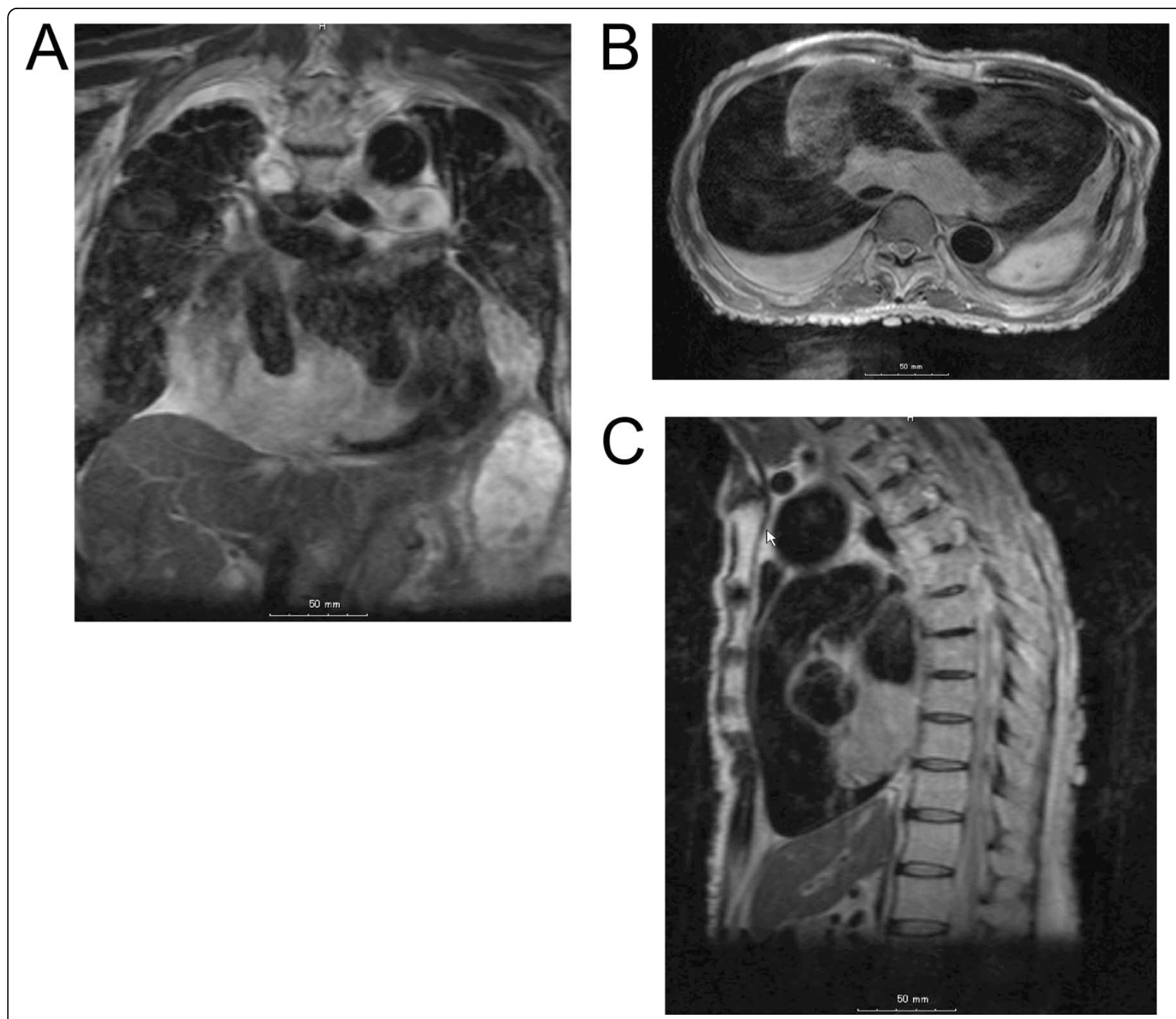

Figure 3 Magnetic resonance imaging showed a large tumor in the inferior-posterior wall of the heart. (A) Frontal cross section. Size: 7.5 $\mathrm{cm}$. (B) Horizontal cross section. Size: $9 \mathrm{~cm}$. (C) Sagittal cross section. Size: $6.5 \mathrm{~cm}$.

of metastatic features of MPNST, however, is still unknown.

The prognosis of patients with MPNST is generally poor. Aggressive surgery significantly improved diseasefree survival [5,8]. Adjuvant chemotherapy and radiotherapy has not been proven to prolong patient survival, but it is effective as a palliative option $[4,8,9]$. If clinical symptoms of cardiac dysfunction occur during the progression of MPNST, it might be that the heart is involved. In such cases of MPNST, it is necessary to exclude cardiac involvement, even if it is rare, by occasional echocardiography. Early diagnosis can allow timely surgical intervention, if the patient is operable, which may improve results, as in the case described here.

Clinical features vary according to the site of cardiac involvement[10], such as pericardium, epicardium, myocardium or endocardium. The present case showed a large mass in the myocardial region, which was accompanied with increasing pericardial effusion and arrhythmia. Serial histological sections revealed that the metastatic tumor markedly affected the common bundle of His, in addition to the ordinary myocardium. Based on these findings, we surmised that complete atrioventricular block was attributable to cardiac metastasis of MPNST, which is causative of circulatory failure [10]. Related features of cardiac involvement of MPNST are uncertain. More cases should be reported to elucidate the clinical entity associated with cardiac involvement of MPNST and to formulate an appropriate treatment strategy.

Most NF1 patients carry a constitutional mutation of the NF1 tumor suppressor gene [11]. Biallelic 


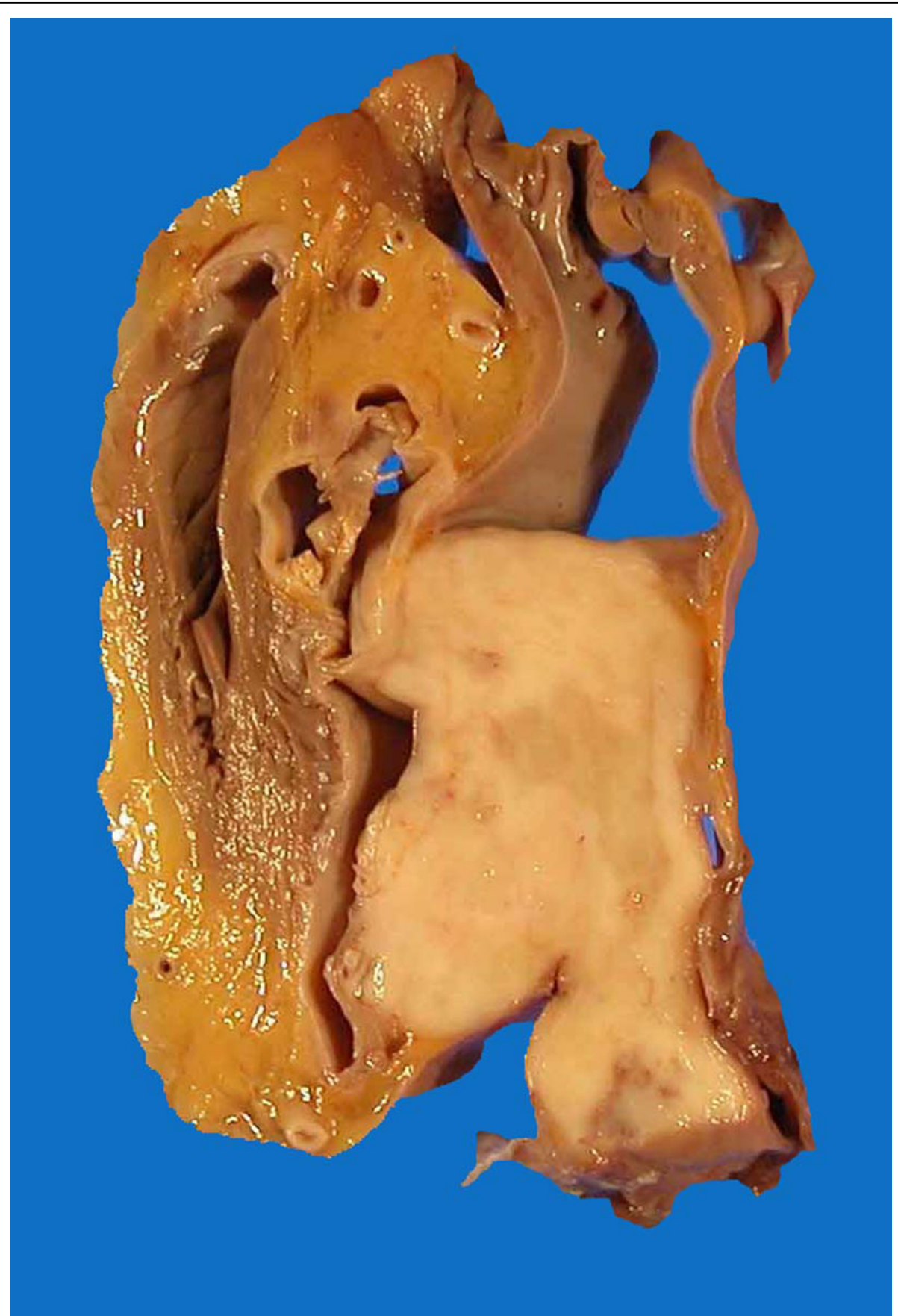

Figure 4 Autopsy specimen showing a tumor in the heart.

inactivation of NF1 and mutations of numerous additional tumor suppressor genes within the $p 19^{A R F}$ $M D M 2-T P 53$ and $p 16^{I N K 4 A_{-}}-R b$ signaling cascades have been identified in MPNSTs [12,13]. These abnormalities of suppressor genes, except for NF1, are not present in neurofibromas. It is therefore thought that the development of neurofibromas and their subsequent progression to become MPNSTs involves a sequential series of tumor suppressor mutations. Deletions and other mutations that result in loss of function of the TP53 tumor suppressor gene are some of the more common abnormalities found in MPNSTs. Biallelic inactivation of the TP53 locus is found rarely in MPNST, which has led to the suggestion that hemizygous TP53 mutations may suffice for neurofibromas to progress and become MPNSTs. 
A recent study has demonstrated that two MPNST cell lines derived from sporadically occurring MPNSTs have functional and intact NF1 genes [14]. Paradoxically, however, microarray studies that have compared the transcriptomes of sporadic and NF1-associated MPNSTs have not found a molecular signature that distinguishes these neoplasms $[14,15]$.

For understanding of these complex neoplasms and the development of the effective new therapy, further investigation will be needed into the clinical features and the basic science.

\section{Conclusion}

Although cardiac involvement of MPNST is rare, precise examination including occasional echocardiography is necessary when clinical signs of tumor development in the heart are suspected.

\section{Consent}

Written informed consent was obtained from the patient's family for publication of this case report and any accompanying images. A copy of the written consent is available for review by the Editor-in-Chief of this journal.

\section{Acknowledgements}

The authors thank Ms. Y. Yamaguchi, Mr. J. Ashimura, Mr. K. Nakagawa, Ms. R. Kitazume, and Mr. K. Yoshizato for technical assistance.

\section{Author details}

${ }^{1}$ Department of Pathology, Osaka Medical Center for Cancer and

Cardiovascular Diseases, 1-3-3 Nakamichi, Higashinari, Osaka, 537-8511, Japan. ${ }^{2}$ Department of Pathology, Osaka University Graduate School of Medicine, 22 Yamadaoka, Suita, Osaka, 565-0871, Japan. ${ }^{3}$ Department of Orthopedic surgery, Osaka Medical Center for Cancer and Cardiovascular Diseases, 1-3-3 Nakamichi, Higashinari, Osaka, 537-8511, Japan. ${ }^{4}$ Department of Life Science, Okayama University of Science, 1-1 Ridaicho, Kita, Okayama, Okayama, 7000005, Japan.

\section{Authors' contributions}

MK participated in the pathological examination of the case, the design of the study and drafting the manuscript.

$\mathrm{NW}, \mathrm{SN}, \mathrm{Nl}$ and $\mathrm{CY}$ participated in the pathological examination of the case. YFJ, MT, and MY participated in the immunohistochemical analysis.

NN and NA participated in collecting clinical data and images.

YT participated in its design and coordination and helped to draft the manuscript.

All authors have read and approved the final manuscript.

\section{Competing interests}

The authors declare that they have no competing interests.

Received: 11 September 2009

Accepted: 9 January 2010 Published: 9 January 2010

\section{References}

1. Weiss SW, Goldbrum JR: Malignant tumors of peripheral nerves. Enzinger and Weiss's Soft Tissue Tumors St Louis: Mosby 2008.

2. Hussain R, Neligan MC: Metastatic malignant schwannoma in the heart. Ann Thorac Surg 1993, 56:374-375.

3. Menezes JAS, Greco OT, Fiorini M, et al: Malignant schwannoma metastasizing to the heart. Arq Bras Cardiol 1992, 58:35-39.
4. Sordillo PP, Helson L, Hajdu SI, et al: Malignant schwannoma-clinical characteristics, survival, and response to therapy. Cancer 1981, 47:25032509.

5. Ducatman BS, Scheithauer BW, Piepgras DG, et al: Malignant peripheral nerve sheath tumors. A clinicopathologic study of 120 cases. Cancer 1986, 57:2006-2021.

6. Schmidt $H$, Würl $P$, Taubert $H$, et al: Genomic imbalances of $7 p$ and $17 q$ in malignant peripheral nerve sheath tumors are clinically relevant. Genes Chromosomes Cancer 1999, 5:205-211.

7. Koga $T$, Iwasaki $H$, Ishiguro $M$, et al: Frequent genomic imbalances in chromosomes 17,19 , and $22 q$ in peripheral nerve sheath tumours detected by comparative genomic hybridization analysis. J Pathol 2002, 197:98-107.

8. Wanebo JE, Malik JM, VandenBerg SR, et al: Malignant peripheral nerve sheath tumors. A clinicopathologic study of 28 cases. Cancer 1993, 71:1247-1253.

9. Wong WW, Hirose T, Scheithauer BW, et al: Malignant peripheral nerve sheath tumor: analysis of treatment outcome. Int J Radiat Oncol Biol Phys 1998, 42:351-360.

10. MacGee W: Metastatic and invasive tumours involving the heart in a geriatric population: a necropsy study. Virchows Archiv A Pathol Anat 1991, 419:183-189.

11. Messiaen LM, Callens T, Mortier G, et al: Exhaustive mutation analysis of the NF1 gene allows identification of $95 \%$ of mutations and reveals a high frequency of unusual splicing defects. Hum Mutat 2000, 15:541-555.

12. Mantripragada KK, Spurlock G, Kluwe $L$, et al: High-resolution DNA copy number profiling of malignant peripheral nerve sheath tumors using targeted microarray-based comparative genomic hybridization. Clin Cancer Res 2008, 14:1015-1024.

13. Birindelli S, Perrone F, Oggionni M, et al: Rb and TP53 pathway alterations in sporadic and NF1-related malignant peripheral nerve sheath tumors. Lab Invest 2001, 81:833-844.

14. Miller SJ, Rangwala F, Williams J, et al: Large-scale molecular comparison of human schwann cells to malignant peripheral nerve sheath tumor cell lines and tissues. Cancer Res 2006, 66:2584-2591.

15. Holtkamp N, Reuss DE, Atallah I, et al: Subclassification of nerve sheath tumors by gene expression profiling. Brain Pathol 2004, 14:258-264.

doi:10.1186/1746-1596-5-2

Cite this article as: Kitamura et al:: Malignant peripheral nerve sheath tumor associated with neurofibromatosis type 1, with metastasis to the heart: a case report. Diagnostic Pathology 2010 5:2.
Publish with Bio Med Central and every scientist can read your work free of charge

"BioMed Central will be the most significant development for disseminating the results of biomedical research in our lifetime. "

Sir Paul Nurse, Cancer Research UK

Your research papers will be:

- available free of charge to the entire biomedical community

- peer reviewed and published immediately upon acceptance

- cited in PubMed and archived on PubMed Central

- yours - you keep the copyright
BioMedcentral 